Pacific Journal of Mathematics

A MEAN STIELTJES TYPE INTEGRAL 


\section{A MEAN STIELTJES TYPE INTEGRAL}

\section{B. Priest}

For the extended mean Stieltjes integral R. A. Stokes has shown that joint discontinuities of the functions involved can be ignored just as in the ordinary mean Stieltjes integral as considered by Porcelli and others. A Stieltjes type integral of a function with respect to a function pair has been defind by $E$. D. Roach, but existence of the integral depends upon the simultaneous continuity of two or more of the functions involved. In this paper a mean Stieltjes type integral of a function with respect to a function pair is defined which overcomes these limitations. Representation theorems for the integral are also given.

1. Definitions and notations. Unless otherwise stated or implied, functions considered in this paper are real valued and defined on the rectangular interval $A=[\alpha, \gamma] \times[\beta, \delta]$. Limits are of the $\sigma$ or refinement type on partitions $D$ of $A[4,10]$. If the subinterval $d=[p, r] \times$ $[q, s]$ of $A$ is an element of the partition $D$, then the $f$-area of the function $f$ is given by $f(d)=f(p, q)-f(r, q)+f(r, s)-f(p, s)$ [6]. The horizontal and vertical contour maps of $f$ are given by $f[-, n]$ and $f[m,-]$, respectively, where $(m, n) \in A$. The function $f$ is of bounded variation in the sense of Vitali provided the sums of the type $\sum_{d \in D}|f(d)|$ are uniformly bounded. $\mathrm{V}_{[p, q ; r, s]}(f)$ (or $V_{d}(f)$ ) will denote the variation of $f$ over $d$. Hardy and Krause require additionally that at least one horizontal and at least one vertical contour map of $f$ be of bounded variation $[1,2]$. The function $f$ is said to be

(1) totally nondecreasing provided $f(d) \geqq 0$ for each $d \in D, f[\alpha$, $-]$ is nondecreasing, and $f[-, \beta]$ is nondecreasing;

(2) partially nondecreasing provided $f(d) \geqq 0$ for each $d \in D$, $f[\alpha,-]$ is nondecreasing, and $f[-, \delta]$ is nonincreasing; and

(3) anchored provided $f(d) \geqq 0$ for each $d \in D$ and $f[\alpha,-]=$ $f[-, \beta]=0[2,11]$.

The $(g, h)$-evaluation over $d \in D$, denoted by $(g, h)(d)$, of the function pair $(g, h)$ is given by $(g(r, q)-g(p, q))(h(p, s)-h(p, q))-(h(r$, $s)-h(p, s))(g(r, s)-g(r, q))$. The lower $(g, h)$-evaluation, denoted by $(g, h)(1, d)$ is given by an analogous expression wherein $g(r, s)-g(r$, $q)$ has been replaced by $g(p, s)-g(p, q) . \quad N_{D}(g, h)$ and $L_{D}(g, h)$ denote the sums $\sum_{d \in D}(g, h)(d)$ and $\sum_{d \in D}(g, h)(1, d)$, respectively. If $E \geqq D$ (i.e., $E$ is a refinement of $D$ ), then $E / d$ denotes that portion of $E$ which is a partition of $d$. Let $N^{\prime}(d)=\sup \left\{N_{E}(g, h): E\right.$ is any partition of $d\} . \quad L^{\prime}(d)$ is defined similarly.

The function $f$ is quasicontinuous [8] provided the limits $f\left(s, t^{ \pm}\right)$, 
$f\left(s^{ \pm}, t\right)$ and $f\left(s^{ \pm}, t^{ \pm}\right)$exist for each $(s, t)$ in $A[12$, p. 3]. The boundary of $d$ will be denoted by $\partial(d)$.

2. The integrator pair. In this section, unless otherwise stated, we assume that $g$ is totally nondecreasing and $h$ is partially nondecreasing. Under these conditions, it follows directly from the definitions that (1) $N^{\prime}(d)$ and $L^{\prime}(d)$ are finite for each $d \in D$; (2) sums of the type $L_{D}(g, h)$ are nondecreasing under refinement; and (3) $L^{\prime}(d)=$ $\sum L^{\prime}(e)$ where the summation is over all $e$ in $E / d$. Thus using techniques analogous to those developed in [12, pp. 10-14] for functions of bounded variation in the sense of Vitali we have the following two lemmas:

Lemma 2.1. Let $\varepsilon>0$ and $d=[p, r] \times[q, s] \leqq A$. Then there exists a pairwise, disjoint collection $\left\{c_{j}\right\}_{1 \leqq j \leqq 4}$ of subintervals of $d$ such that

(1) each $c_{j}$ shares a separate vertex with $d$ and

(2) if $c_{j}$ is a subinterval of $c_{j}$ which also shares a vertex with $d$, then $L^{\prime}\left(c_{j}\right)-L^{\prime}\left(c_{j}^{\prime}\right)<\varepsilon$.

LEMma 2.2. Let $\varepsilon>0, d=[p, r] \times[q, s] \cong A$, and $b$ be a subinterval of $d$ missing the vertices of $d$ such that $\partial(d) \cap \partial(b) \neq \varnothing$. Then there exists a proper subinterval $b^{\prime}$ of $b$ such that if $E$ is a partition of $b^{\prime}$, then $\sum L^{\prime}(e)<\varepsilon$ where the summation is over all $e$ in $E$ which have no point in common with $\partial(d)$.

LEMma 2.3. If $g[-, \delta]$ or $h[-, \beta]$ is continuous on $[\alpha, \gamma]$, then for each $\varepsilon>0$, there is a partition $D$ of $A$ such that $0 \leqq N_{E}(g, h)-$ $L_{E}(g, h)<\varepsilon$ where $E \geqq D$.

Proof. Assume that $g[-, \delta]$ is continuous. If $h[-, \beta]$ is continuous, the proof is similar. Let $\varepsilon>0$. Assume that $K=h(\alpha, \beta)-$ $h(\gamma, \beta) \neq 0$; otherwise, the result is immediate. There exists a partition $D=\left\{d_{i}=\left[t_{i-1}, t_{i}\right] \times[\beta, \delta]\right\}_{1 \leqq i \leqq n}$ of $A$ such that $g\left(d_{i}\right)<\varepsilon / K$. Let $E$ be a refinement of $D$. Under the conditions on $g$ and $h$, it follows routinely that

$$
N_{E}(g, h)-L_{E}(g, h) \leqq \sum_{i=1}^{n}\left(h\left(t_{i-1}, \beta\right)-h\left(t_{i}, \beta\right)\right)\left(g\left(d_{i}\right)\right)<\varepsilon .
$$

That the difference $N_{E}(g, h)-L_{E}(g, h)$ is nonnegative follows readily from the definitions.

LEMMA 2.4. If $f$ is a function of bounded variation in the sense of Vitali and each of its horizontal contour maps is continuous, then the variation function $V(x)=V_{[\alpha, \beta ; x, \delta]}(f)$ for each $x$ in $[\alpha, \gamma]$, is 
continuous.

The following theorem parallels a known result concerning functions of bounded variation in Vitali's sense [9, p. 250].

THEOREM 2.5. If the function $g$ is of bounded variation in the sense of Hardy-Krause, then there exist totally nondecreasing functions $g_{1}$ and $g_{2}$ and partially nondecreasing functions $h_{1}$ and $h_{2}$ such that $g=g_{2}-g_{1}=h_{2}-h_{1}$. Moreover, if the horizontal contour maps of $g$ are continuous, then $g_{1}[-, \delta], g_{2}[-, \delta], h_{1}[-, \beta]$, and $h_{2}[-, \beta]$ are continuous.

Proof. It is known that if $g$ is of bounded variation in the sense of Hardy-Krause, then $g[\alpha,-], g[-, \beta]$, and $g[-, \delta]$ are of bounded variation in the usual sense $[2, \mathrm{p} .385]$. Thus let $g_{1}$ be the function such that

$$
g_{1}(x, y)=V_{[\alpha, \beta ; x, y]}(g)+V_{\alpha}^{x}(g[-, \beta])+V_{\beta}^{y}([\alpha,-])
$$

for all $(x, y)$ in $A$. Let $h_{1}$ be such that

$$
h_{1}(x, y)=V_{[\alpha, \beta ; x, y]}(g)-V_{[\alpha, \beta ; x, \delta]}(g)+V_{\beta}^{y}(g[\alpha,-])-V_{. \alpha}^{x}(g[-, \delta])
$$

for all $(x, y)$ in $A$. Additionally let $g_{2}=g+g_{1}$ and $h_{2}=g+h_{1}$. The theorem now follows from the additivity of the variation functions on subintervals of a given interval [5, p. 107] and Lemma 2.4.

3. The integral. For the remainder of this paper, $F(d)$ will denote $1 / 4(f(p, q)+f(r, q)+f(r, s)+f(p, s))$, the mean evaluation of $f$ at the four vertices of $d$ in the partition $D$. [4, p. 274 and 12, p. 5]

Definition 3.1. The function $f$ is $(g, h)$-integrable provided the $\sigma$-limit of sums of the type $S_{D}(f, g, h)=\sum_{d \in D}(F(d))((g, h)(d))$ exists. The integral will be denoted by $\int_{A} f d g d h$.

REMARKS. It follows immediately from the definition that the integral is linear in each of its three components i.e., the integrand $f$ and each of its integrator function $g$ and $h$. Also, if each of $g$ and $h$ is a function of a single variable, then $\int_{A} f d g d h$ agrees readily with one or the other of the extended mean Stieltjes integrals $\pm m(\sigma) \int_{A} f d k$ where $k=g h$. For in this case, $\lim _{\sigma} S_{D}(f, g, h)=\lim _{\sigma} \pm$ $\sum_{d \in D} F(d) k(d)= \pm m(\sigma) \int_{A} f d k[12$, p. 14]. 
THEOREM 3.2. If $f$ is quasicontinuous, $g$ and $h$ are of bounded variation in the sense of Hardy-Krause, and either the horizontal contour maps of $g$ or the horizontal contour maps of h are continuous, then $f$ is $(g, h)$-integrable.

Proof. Let $\varepsilon>0$. In view of Theorem 2.5 and the remarks preceding this theorem, it suffices to consider a totally nondecreasing $g$ and a partially nondecreasing $h$ such that either $g[-, \delta]$ or $h[-$, $\beta]$ is continuous. There is a partition $A^{\prime}=\left\{A_{1}, \cdots, A_{N}\right\}$ of $A$ such that (1) $\left|L_{E}(g, h)-L_{F}(g, h)\right|<\varepsilon$ where $E, F \geqq A^{\prime}$ and (2) if $P$ and $Q$ are in the interior of $A_{i}$ or in the interior of a particular edge of $A_{i}$ for some $i \leqq N$, then $|f(P)-f(Q)|<\varepsilon[12$, p. 4]. By Lemma 2.1 and 2.2 there exists a refinement $B$ of $A^{\prime}$ such that $B / A_{i}=\left\{c_{i j}\right.$, $\left.e_{i j}, I_{i}\right\}_{1 \leqq j \leqq 4}$ where each $c_{i j}$ has the property of $c_{j}$ in Lemma 2.1, each $e_{i j}$ has the property of $b^{\prime}$ in Lemma 2.2 and $\left.I_{i}=A_{i}\right) \bigcup_{j=1}^{4}\left(c_{i j} \cup e_{i j}\right)$, the complement of $\bigcup_{j=1}^{4}\left(c_{i j} \cup e_{i j}\right)$ relative to $A_{i}$. Suppose $G \geqq B$ and let $C_{i j}=\left\{e \in G / c_{i j} \mid e\right.$ shares a vertex with $\left.A_{i}\right\}, E_{i j}=\left\{e \in G / e_{i j} \mid \partial(e) \cap\right.$ $\left.\partial\left(A_{i}\right) \neq \varnothing\right\}, C_{i j}^{\prime}=\left(G / c_{i j}\right) \backslash C_{i j}$, and $E_{i j}^{\prime}=\left(G / e_{i j}\right) \backslash E_{i j}$. Then

$$
\begin{aligned}
& \left|\sum_{b \in B}(F(b))((g, h)(1, b))-\sum_{e \in G}(F(e))((g, h)(1, e))\right|<M\left|L_{B}(g, h)-L_{G}(g, h)\right| \\
& \quad+\varepsilon\left[\sum_{i=1}^{N} \sum_{j=1}^{4}\left(L_{C_{i j}}(g, h)+L_{E_{i j}}(g, h)\right)\right]+\varepsilon \sum_{i=1}^{N} L_{G / I_{i}}(g, h) \\
& \quad+2 M\left[\sum_{i=1}^{N} \sum_{j=1}^{4} \sum_{e \in E^{\prime}} L_{i j}^{\prime}(e)+\sum_{i=1}^{N} \sum_{j=1}^{4} \sum_{e \in C_{i j}^{\prime}} L^{\prime}(e)\right]<\varepsilon K
\end{aligned}
$$

where $M$ is a positive bound on $f\left[12\right.$, p. 4] and $K=M+L^{\prime}(A)+$ $16 M N$. Now by Lemma 2.3 there is a partition $B^{\prime}$ of $A$ such that if $G \geqq B^{\prime}, 0 \leqq N_{G}(g, h)-L_{G}(g, h)<\varepsilon / 2 M$. Suppose $D$ refines $B$ and $B^{\prime}$. It now follows routinely that $\left|S_{E}(f, g, h)-S_{F}(f, g, h)\right|<\varepsilon(2 K+$ $1)$, where $E, F \geqq D$. It is well known that this Cauchy condition insures the $\sigma$-limit since $S_{D}(f, g, h)$ is a function of subdivisions [4, Th. 2.11, p. 266] i.e., $f$ is $(g, h)$-integrable.

4. Representations for the integral. We now develop certain representation theorems for the integral, establishing relationships between it, the ordinary mean Stieltjes integral $\left(m(\sigma) \int_{\alpha}^{\gamma} f d g[3,10]\right)$, and the extended mean Stieltjes integral. We shall say that $f$ is factorable into functions $f_{1}$ and $f_{2}$ provided there exist functions $f_{1}$ and $f_{2}$ such that $f(x, y)=f_{1}(x) f_{2}(y)$ for each $(x, y)$ in $A$.

THEOREM 4.1. Suppose $f$ is quasicontinuous and $g$ and $h$ are of bounded variation in the sense of Vitali and factorable into nonconstant functions $g_{1}, g_{2}$, and $h_{1}, h_{2}$ respectively. If $\mu=h_{1} g_{2}$ and $\nu=g_{1} h_{2}$ have 
no common discontinuities, then $f$ is $(g, h)$-integrable and $\int_{A} f d g d h=$ $m(\sigma) \int_{A} f \mu d \nu-m(\sigma) \int_{A} f \nu d \mu$.

Proof. Since $g$ and $h$ are of bounded variation in Vitali's sense and factorable into nonconstant functions, $\mu$ and $\nu$ are of bounded variation in Vitali's sense [5, p. 107]. It also follows readily that $f \mu$ and $f \nu$ are quasicontinuous [5, p. 40]. Thus each of $m(\sigma) \int_{A} f \mu d \nu$ and $m(\sigma) \int_{A} f \nu d \mu$ exists [12, Th. 3.1, p. 15]. Since $S_{D}(f, g, h)=$ $\sum_{d \in D}(F(d))(\mu(p, q))(\nu(d))-\sum_{d \in D}(F(d))(\nu(r, s)(\mu(d))$, the result follows by showing that the $\sigma$-limits of the sums on the right are $m(\sigma) \int_{A} f \mu d \nu$ and $m(\sigma) \int_{A} f \nu d \mu$ respectively. An outline of the proof of the first of these is given. Suppose $V=V_{A}(\nu)>0$. Let $J, K$ and $L$ denote positive bounds on $f, g_{2}$ and $h_{1}$ respectively and let $\varepsilon>0$. There is a partition $A^{\prime}=\left\{A_{1}, \cdots, A_{N}\right\}$ of $A$ such that if $P$ and $Q$ are in the interior of $A_{i}$ for some $i,|\mu(P)-\mu(Q)|<\varepsilon / 4 J V$; and if $E \geqq A^{\prime}, \mid m(\sigma) \int_{A} f \mu d \nu-$ $\sum_{e \in E} F M(e) \nu(e) \mid<\varepsilon / 4$ [12, Th. 3.1, p. 15]. Under the continuity conditions on $\mu$ and $\nu$, there is a refinement $B$ of $A^{\prime}$ such that $B / A_{i}=$ $\left\{c_{i j}, e_{i j}, I_{i}\right\}_{1 \leqq j \leqq 4}$ and such that either (1) $V_{b}(\nu)<\varepsilon / 64 J K L N$ (for $b=$ $c_{i j}$ or $\left.e_{i j}\right)$ or (2) $|\mu(u, v)-\mu(s, t)|<\varepsilon / 8 J V$ where $E \geqq B$ and $(s, t) \epsilon$ $[u, w] \times[v, x] \in E / b$ (for $b=c_{i j}$ or $e_{i j}$ ). Thus if $E \geqq B$ and $b$ ranges over all $c_{i j}$ and $e_{i j}$,

$$
\begin{aligned}
\mid m(\sigma) \int_{A} f \mu d \nu & -\sum_{e \in E}(F(e))(\mu(u, v))(\nu(e)) \mid<\frac{\varepsilon}{4} \\
& +\sum_{e \in E / b}|F M(e)-F(e)(\mu(u, v))||\nu(e)| \\
& +\sum_{i=1}^{N} \sum_{e \in E^{\prime} / I_{i}}|F M(e)-F(e)(\mu(u, v))||\nu(e)| \\
< & \frac{\varepsilon}{4}+\frac{\varepsilon}{2}+\frac{\varepsilon}{4}=\varepsilon .
\end{aligned}
$$

Similarly one can see that $\lim _{\sigma} \sum_{d \in D}(F(d))(\nu(r, s))(\mu(d))=m(\sigma) \int_{A} f \nu d \mu$. Thus $\lim _{\sigma} S(f, g, h)=m(\sigma) \int_{A} f \mu d \nu-m(\sigma) \int_{A} f \nu d \mu$.

REMARK. If in the above theorem we were to allow $f$ to be a nonconstant factorable function and require only that $g_{i}$ and $h_{i}(i=$ $1,2)$ share no discontinuities from the right or from the left, then we can show by a similar argument that the integral $\int_{A} f d g d h$ can 
be represented by

$$
\left(m(\sigma) \int_{\alpha}^{r} f_{1} h_{1} d g_{1}\right)\left(m(\sigma) \int_{\beta}^{\delta} f_{2} g_{2} d h_{2}\right)-\left(m(\sigma) \int_{\alpha}^{\gamma} f_{1} g_{1} d h_{1}\right)\left(m(\sigma) \int_{\beta}^{\delta} f_{2} h_{2} d g_{2}\right) .
$$

THEOREM 4.2. Suppose $f$ is quasicontinuous and $g$ and $h$ are of bounded variation in the sense of Hardy-Krause. If the contour maps of $g[$ resp. $h]$ are continuous, then there exists a function $\mu$ of bounded variation such that $\int_{A} f d g d h=m(\sigma) \int_{A} f d \mu$.

Proof. By Theorem 3.2 $\int_{A} f d g d h$ exists. Let $\mu$ be such that for each $(x, y) \in A$,

$$
\mu(x, y)=m(\sigma) \int_{0}^{x} h[-, y] d g[-, y]+m(\sigma) \int_{0}^{y} g[x,-] d h[x,-]-(g h)(x, y) .
$$

By Theorem 2.5, it suffices to consider $g$ and $h$ as in Theorem 3.2. Under these conditions and known integration by parts formulas [7, Th. 2.1, p. 61] it follows that $\sum_{d \in D} \mu(d)$ is equal to

$$
\begin{aligned}
& \sum_{d \in D}(g(r, q)-g(p, q))(h(\lambda, s)-h(\lambda, q)) \\
& -\sum_{d \in D}(h(r, \theta)-h(p, \theta))(g(r, s)-g(r, q)) \\
& -\sum_{d \in D}\left(h\left(\lambda^{\prime}, s\right)-h\left(p, \theta^{\prime}\right)\right)(g(d))
\end{aligned}
$$

where $d=[p, r] \times[q, s], \lambda, \lambda^{\prime} \in[p, r]$, and $\theta, \theta^{\prime} \in[q, s]$. Whereupon it follows that $\mu$ is of bounded variation in the sense of Vitali and hence that $m(\sigma) \int_{A} f d \mu$ exists [12, Th. 3.1, p. 15]. Since the last term of $\sum_{d \in D} \mu(d)$ tends to zero under refinement, it follows that $\lim _{\sigma} \sum_{d \in D} F(d) \mu(d)=\int_{A} f d g d h$ i.e., $m(\sigma) \int_{A} f d \mu=\int_{A} f d g d h$.

THEOREM 4.3. Suppose $f$ is quasicontinuous and $g$ and h are of bounded variation in the sense of Hardy-Krause. If the horizontal and vertical contour maps of $h$ [resp.g] are continuous, then there exist function sequences $\left(h_{1_{p}}\right),\left(h_{2_{p}}\right),\left(k_{1_{p}}\right)$, and $\left(k_{2_{p}}\right)$ such that

$$
\int_{A} f d g d h=\sum_{p=1}^{\infty}\left(\int_{A} f d h_{1_{p}} d k_{1_{p}}\right)-\sum_{p=1}^{\infty}\left(\int_{A} f d h_{2_{p}} d k_{2_{p}}\right) .
$$

Proof. By Theorem 4.2 there is a function $\mu$ such that $\int_{A} f d g d h=$ $m(\sigma) \int_{A} f d \mu$. As in that theorem, it can be shown that $\mu$ is bounded variation in the sense of Hardy-Krause. By Theorem 2.5, $\mu$ is the difference of two nodecreasing functions $F_{1}$ and $F_{2}$. By a theorem 
of Jolly [6, Th. 1, p. 317] there exist anchored nondecreasing functions $G_{1}$ and $G_{2}$, nondecreasing functions $g_{1}$ and $g_{2}$ on $[\alpha, \gamma]$, and nondecreasing functions $h_{1}$ and $h_{2}$ on $[\beta, \delta]$ such that $F_{i}=G_{i}+g_{i}+h_{i}$ (for $i=$ 1, 2). Thus $\int_{A} f d g d h=m(\sigma) \int_{A} f d G_{1}-m(\sigma) \int_{A} f d G_{2}$. By a theorem of Stokes [12, Th. 4.3, p. 34] there exist function sequences $\left(h_{1_{p}}\right)$ and $\left(h_{2_{p}}\right)$ each term of which is defined on $[\alpha, \gamma]$ and function sequences $\left(k_{1_{p}}\right)$ and $\left(k_{2_{p}}\right)$ each term of which is defined on $[\beta, \delta]$ such that

$$
\sum_{p=1}^{n} \int_{A} f d\left(h_{1_{p}} k_{1_{p}}\right) \longrightarrow \int_{A} f d G_{1}
$$

and

$$
\sum_{p=1}^{n} \int_{A} f d\left(h_{2_{p}} k_{2_{p}}\right) \longrightarrow \int_{A} f d G_{2}
$$

as $n \rightarrow \infty$. Since each $h_{i_{p}}$ is a function of $x$ and each $k_{i_{p}}$ is a function of $y$, the theorem now follows from the remarks preceding Theorem 3.2.

\section{REFERENCES}

1. C. R. Adams and J. A. Clarkson, On definitions of bounded variation for functions of two variables, Trans. Amer. Math. Soc., 35 (1933), 824-854.

2. B. J. Baker, Concerning separation of variables, J. London Math. Soc., 42 (1967), 385-388.

3. R. C. Bzoch, On the existence of Stieltjes integrals, Portugal. Math., 18 (1959), 121-124.

4. T. H. Hildebrant, Definitions of Stieltjes integrals of the Riemann type, Amer. Math. Monthly, 45 (1938), 265-277.

5. - Introduction to the Theory of Integration, Academic Press, New York, 1963.

6. R. F. Jolly, On the representation of simple surfaces, J. London Math. Soc., 42 (1967), 316-320.

7. R. E. Lane, The integral of a function with respect to a function, Proc. Amer. Math. Soc., 5 (1954), 59-66.

8. Linear operators on quasi-continuous functions, Trans. Amer. Math. Soc., 89 (1958), 378-394.

9. E. J. McShane, Integration, Princeton University Press, Princeton, 1944.

10. P. Porcelli, On the existence of the Stieltjes mean- $\sigma$-integral, Illinois J. Math., 2 (1958), 124-128.

11. E. D. Roach, An integral, Dissertation, University of Texas (1965).

12. R. A. Stokes, The extended mean Stieltjes integral, Lecture notes, University of Mississippi, (1968).

Received August 30, 1971 and in revised form March 24, 1972.

UNIVERSITY OF MISSISSIPPI

AND

HARDING COLLEGE 



\section{PACIFIC JOURNAL OF MATHEMATICS}

\section{EDITORS}

H. SAMELSON

Stanford University

Stanford, California 94305

C. R. Hовву

University of Washington

Seattle, Washington 98105
J. DugundJI

Department of Mathematics University of Southern California

Los Angeles, California 90007

RICHARD ARENS

University of California

Los Angeles, California 90024

\section{ASSOCIATE EDITORS}

E. F. BECKENBACH

B. H. NeumanN

F. WOLF

K. YoSHIDA

\section{SUPPORTING INSTITUTIONS}

UNIVERSITY OF BRITISH COLUMBIA

CALIFORNIA INSTITUTE OF TECHNOLOGY

UNIVERSITY OF CALIFORNIA

MONTANA STATE UNIVERSITY

UNIVERSITY OF NEVADA

NEW MEXICO STATE UNIVERSITY

OREGON STATE UNIVERSITY

UNIVERSITY OF OREGON

OSAKA UNIVERSITY
UNIVERSITY OF SOUTHERN CALIFORNIA

STANFORD UNIVERSITY

UNIVERSITY OF TOKYO

UNIVERSITY OF UTAH

WASHINGTON STATE UNIVERSITY

UNIVERSITY OF WASHINGTON

AMERICAN MATHEMATICAL SOCIETY

NAVAL WEAPONS CENTER

Printed in Japan by International Academic Printing Co., Ltd., Tokyo, Japan 


\section{Pacific Journal of Mathematics}

\section{Vol. 44, No. $1 \quad$ May, 1973}

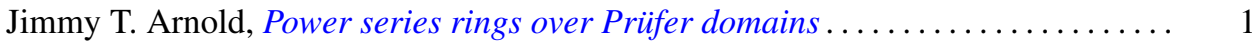

Maynard G. Arsove, On the behavior of Pincherle basis functions . . . . . . . . . 13

Jan William Auer, Fiber integration in smooth bundles ................. 33

George Bachman, Edward Beckenstein and Lawrence Narici, Function algebras

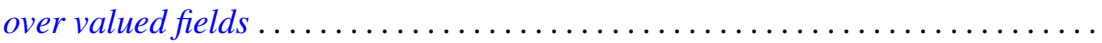

Gerald A. Beer, The index of convexity and the visibility function . . . . . . . . . . .

James Robert Boone, A note on mesocompact and sequentially mesocompact

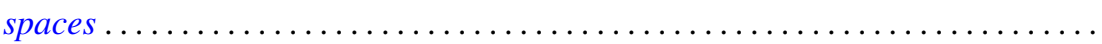

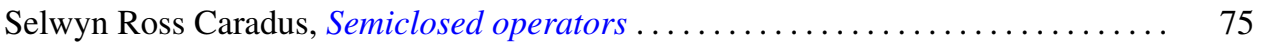

John H. E. Cohn, Two primary factor inequalities . . . . . . . . . . . . . . . 81

Mani Gagrat and Somashekhar Amrith Naimpally, Proximity approach to

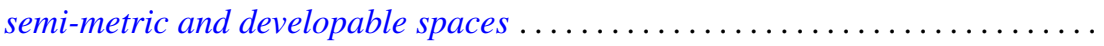

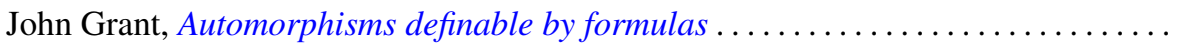

Walter Kurt Hayman, Differential inequalities and local valency ..............

Wolfgang H. Heil, Testing 3-manifolds for projective planes . . . . . . . . . . . . .

107

Melvin Hochster and Louis Jackson Ratliff, Jr., Five theorems on Macaulay

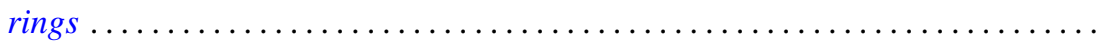

Thomas Benton Hoover, Operator algebras with reducing invariant subspaces ....

James Edgar Keesling, Topological groups whose underlying spaces are separable

Fréchet manifolds...

Frank Leroy Knowles, Idempotents in the boundary of a Lie group . .

191

George Edward Lang, The evaluation map and EHP sequences ...

201

Everette Lee May, Jr, Localizing the spectrum . . . . . . . . . . . .

211

Frank Belsley Miles, Existence of special $K$-sets in certain locally compact abelian groups.

Susan Montgomery, A generalization of a theorem of Jacobson. II . .

T. S. Motzkin and J. L. Walsh, Equilibrium of inverse-distance forces in

three-dimensions.

Arunava Mukherjea and Nicolas A. Tserpes, Invariant measures and the converse

of Haar's theorem on semitopological semigroups .

James Waring Noonan, On close-to-convex functions of order $\beta$

Donald Steven Passman, The Jacobian of a growth transformation

Dean Blackburn Priest, A mean Stieltjes type integral ........ .

Joe Bill Rhodes, Decomposition of semilattices with applications to topological



Claus M. Ringel, Socle conditions for $\mathrm{QF}-1$ rings ..........

Richard Rochberg, Linear maps of the disk algebra

Roy W. Ryden, Groups of arithmetic functions under Dirichlet convolution . .

Michael J. Sharpe, A class of operators on excessive functions

Erling Stormer, Automorphisms and equivalence in von Neumann algebras ..

Philip C. Tonne, Matrix representations for linear transformations on series

analytic in the unit disc. 